\title{
Resonant Excitation in a Low-Pressure Linear Ion Trap
}

\author{
B. A. Collings, W. R. Stott, and F. A. Londry \\ MDS SCIEX, Concord, Ontario, Canada
}

\begin{abstract}
It has been shown that through the process of resonant excitation the fragmentation of ions confined in a low-pressure $(<0.05$ mTorr) linear ion trap (LIT) can be accomplished while maintaining both high fragmentation efficiency and high resolution of excitation. The ion reserpine, $609.23 \mathrm{Da}$, has been fragmented with efficiencies greater than $90 \%$ while a higher mass ion, a homogeneously substituted triazatriphosphorine of mass $2721.89 \mathrm{Da}$, has been fragmented with $48 \%$ efficiency. This was accomplished by extended resonant excitation by low-amplitude auxiliary RF signals. Computer modelling of ion trajectories and analysis of the trapping potentials have demonstrated that a reduction in neutralization of ions on the rods (or losses on the rods) and increased fragmentation is a consequence of higher order terms in the potential introduced by the round-rod geometry of the LIT. (J Am Soc Mass Spectrom 2003, 14, 622-634) (C) 2003 American Society for Mass Spectrometry
\end{abstract}

$\mathrm{R}$ esonance excitation of ions in a linear ion trap (LIT), leading to neutralization on the rods and/or fragmentation, has been demonstrated previously using both quadrupolar and dipolar auxiliary RF signals [1-4]. Typically, these experiments were carried out using nitrogen collision gas, at pressures ranging from 1.5 to $7 \mathrm{mTorr}$, with exposures to resonant excitation of a few milliseconds. At these pressures and exposures it was possible to obtain increased resolutions of excitation from 75 to 250 as the pressure in the LIT was decreased [3]. In those experiments, resolution decreased as the amplitude of the auxiliary signal was increased. However, the auxiliary amplitude required for either neutralization on the rods or fragmentation was dependent upon the pressure of the buffer gas. When the auxiliary amplitude was too low, thermalizing collisions reduced ion energies, both translational and internal, at a rate greater than energy was added by the auxiliary signal. In consequence, there existed a threshold for the auxiliary amplitude below which neutralization/fragmentation did not occur [5, 6]. When the auxiliary amplitude was only slightly above this threshold, resolution of excitation was high, but the degree of neutralization/fragmentation was low [3]. In order to achieve a high degree of neutralization/fragmentation, it was necessary to increase the auxiliary amplitude and suffer the decreased resolution.

The range of frequencies, about the secular frequency, to which ions respond (frequency response) increases with pressure. This behavior is predicted by

Published online May 2, 2003

Address reprint requests to Dr. B. A. Collings, MDS SCIEX, 71 Four Valley Drive, Concord, Ontario L4K 4V8, Canada. E-mail: bruce.collings@ sciex.com the theory of a forced damped harmonic oscillator (FDHO). Models based on the FDHO theory have been used to describe the behavior of ions confined in a Paul trap at pressures in the mTorr regime [7, 8]. However, when excited on-resonance at pressures below one mTorr, it has been assumed generally that ions would be lost on the rods before significant fragmentation occurred. In contrast, it is well known that effective fragmentation at low pressures $\left(1 \times 10^{-4}\right.$ to $1 \times 10^{-6}$ torr) can be achieved through the use of off-resonance excitation, as demonstrated in sustained off-resonance irradiation (SORI) experiments in FT-ICR [9-11].

In the work reported here, ions confined in a LIT (mass range $\mathrm{m} / \mathrm{z} 100$ to 2800 ), were excited resonantly using a dipolar auxiliary signal in a low-pressure $(<0.05$ mTorr) environment. Results show that resolution of fragmentation greater than one thousand for mass 609.23 can be obtained. In addition, high fragmentation efficiency is demonstrated for the ions reserpine (609.23 $\mathrm{m} / \mathrm{z}$ ) and a homogeneously substituted triazatriphosphorine $(2721.89 \mathrm{~m} / \mathrm{z})$. The ability to excite ions with both high resolution of excitation and high fragmentation efficiency is explained with the aid of ion-trajectory simulations and analysis of the higher order terms added to the quadrupolar trapping potential by the round-rod geometry of the LIT.

The ability to fragment ions at pressures typical of the analyzing stage of a triple quadrupole mass spectrometer, allows the analyzing quadrupole to be used in its traditional mode of operation as either a mass filter, or as a LIT with $\mathrm{MS}^{\mathrm{n}}$ capability, combining the quantitative characteristics of a triple quadrupole mass spectrometer and the qualitative characteristics of an ion trap on the same platform [12]. 
Q2

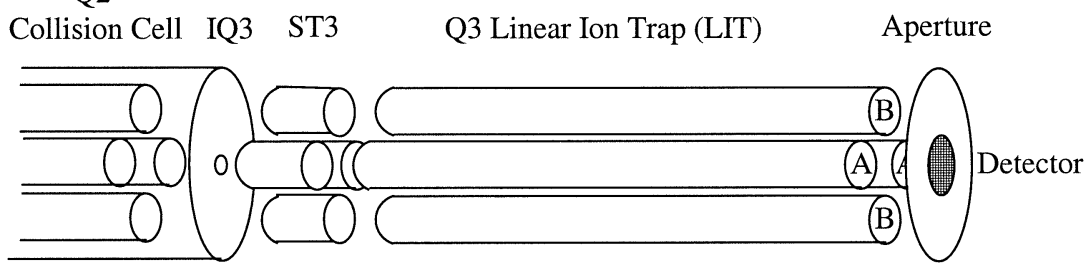

(B)

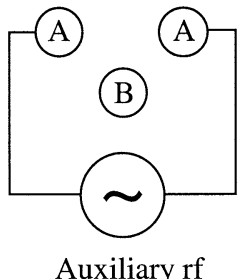

Auxiliary rf

Figure 1. Schematic representation of the LIT. Dipolar excitation was applied across one pair of poles.

\section{Experimental}

Experiments were carried out on a modified triple quadrupole mass spectrometer (API 4000, MDS Sciex, Concord, Ontario, Canada) consisting of an atmospheric pressure ionization (API) source, an atmospheric pressure to vacuum interface, a collisional cooling region containing a radio frequency (RF) only quadrupole $(\mathrm{Q} 0)$ for increased ion transmission and a high vacuum stage. The high vacuum stage contained three quadrupoles in series, an analyzing quadrupole (Q1), a collision cell containing an RF-only quadrupole $(\mathrm{Q} 2)$, and an additional analyzing quadrupole (Q3), which also served as the LIT. Each of the quadrupoles was $200 \mathrm{~mm}$ in length with a field radius of $4.17 \mathrm{~mm}$ and was operated at a drive frequency of $816 \mathrm{kHz}$. The Q3 quadrupole was constructed from round goldcoated ceramic rods. The section of the instrument in which the LIT was located is shown in Figure 1. In these experiments, the mass spectrometer was operated in the LIT mode with the Q1-resolution set to transmit the entire isotopic cluster of the parent ion. Either ions were transmitted through the Q2 collision cell, containing 5-10 mTorr of nitrogen, at low energy, which avoided fragmentation of the parent; or at high energy, which caused the parent ion to fragment through the process of collisionally activated dissociation (CAD). Ions exited the Q2 collision cell through the aperture IQ3, passed through the Brubaker lens ST3 and entered the Q3 quadrupole, which was operated in RF-only mode. Typically, ions were injected into the LIT (Q3) at qvalues ranging from 0.09 to 0.21 . The exit lens was raised to a high DC potential to contain the ions in Q3. Typically the Q3 quadrupole was held at a DC offset potential 5 to $10 \mathrm{~V}$ below that of the Q2 collision cell with the ST3 lens at some intermediate value. While traversing Q3, ions lose sufficient translational energy through collisions with the background nitrogen so that few are lost when they return to the entrance fringing- field after reflecting from the exit lens potential barrier. Typically, the pressure in the vacuum chamber was between $2.7 \times 10^{-5}$ to $4.5 \times 10^{-5}$ torr, measured at the vacuum chamber wall. On average, an ion such as reserpine, with a collision cross-section of $280 \AA^{2}$ with nitrogen [13], will suffer one or more collisions twothirds of the time when travelling twice the length of Q3 (a total distance of $400 \mathrm{~mm}$ ). Even more collisions will be suffered near the exit of the collision cell where the pressure is expected to be higher. After a fill-time of 2 to $50 \mathrm{~ms}$ determined by the ion beam intensity, the potential on the ST3 lens was increased to prevent additional ions from entering the $\mathrm{Q} 3$ quadrupole.

In the LIT, isolation of a specific ion was accomplished by mass-resolving in Q1 and bringing ions into Q2 at sufficiently low axial energy to minimize fragmentation. Alternately, resolving DC can be applied to the Q3 quadrupole to isolate ions of interest in the apex of the first stability region [14]; however, this technique was not used in these experiments.

Using a dipolar auxiliary signal, ions were excited at their fundamental secular frequency

$$
\omega_{0}=\beta \frac{\Omega}{2}
$$

where $\Omega$ is the angular frequency of the RF drive and $\beta$ is a function of the Mathieu stability parameters $a$ and $q$. When $a \ll q$ and $q<0.4$, that function, a continued fraction expression [15], can be approximated with reasonable accuracy by

$$
\beta \approx\left[a+\frac{q^{2}}{2}\right]^{1 / 2}
$$

When no resolving DC is applied to the rods, $a=0$, and eqs 1 and 2 can be combined to obtain an approximation 
of the fundamental secular frequency in terms of the stability parameter $q$ as

$$
\omega_{0} \approx q \frac{\Omega}{2 \sqrt{2}}
$$

Typically, ions were excited at low $q$ by an auxiliary signal supplied by an Agilent 33120A arbitrary waveform generator. To isolate the auxiliary waveforms from the RF drive, the auxiliary signal was coupled to one pole of the LIT through a toroidal bipolar transformer, as shown in Figure 1. This set-up was detailed in reference [1]. A second auxiliary signal, at $312 \mathrm{kHz}$, used for the purpose of mass selective axial ejection (MSAE) [Londry, F. A.; Hager, J. W. Mass-Selective Axial Ejection (in preparation)] at $q=0.846$, was supplied by a module constructed in-house. Using MSAE, ions were scanned out of the LIT at $1000 \mathrm{Da} / \mathrm{s}$.

Frequency response curves were obtained by collecting mass spectra as a function of auxiliary frequency. Spectra were collected at regular intervals over a range that spanned the resonance. In the work presented here, ions were excited resonantly with the RF amplitude, $V_{R F} \approx 150$ $\mathrm{V}$, corresponding to $q \approx 0.21$ for $\mathrm{m} / \mathrm{z} 609$. This setting offered a good compromise between a radial potential well, sufficiently deep to reduce losses from scattering [16, 17], while retaining an acceptable mass range of fragment ions. That is, the lowest stable mass is determined by the ratio of the $q$, which corresponds to the auxiliary frequency, and the low-mass cut-off at $q=0.908$. In this case, fragment ions whose mass was less than 0.23 times that of the 609 Da parent would fall outside the region of stability.

\section{Ion-Trajectory Simulations}

Ion trajectories were simulated using a modelling programme developed in-house [simulator code written by F. A. Londry]. These were used to study the response of ions to an auxiliary dipolar signal. The differences in the behavior of ions in pure-hyperbolic and round-rod quadrupolar fields illustrate how the higher-order terms (above quadrupolar), introduced by the round-rod geometry, resulted in fewer losses on the rods and increased fragmentation in a low-pressure environment. Inelastic collisions with nitrogen gas were simulated using the hard sphere model. The initial starting coordinates, initial thermal kinetic energy, time between collisions, scattering angle, and initial RF phase were all chosen randomly. To limit the size of the trajectory files spanning $50 \mathrm{~ms}$ to a manageable number of points, output was averaged over intervals of two RF cycles. Consequently, micromotion is not visible in the trajectory plots.

Each ion trajectory was calculated incrementally, twenty times each RF cycle, for $50 \mathrm{~ms}$, or until the ion was neutralized on a rod, by integrating the equation of motion. For a singly charged positive ion

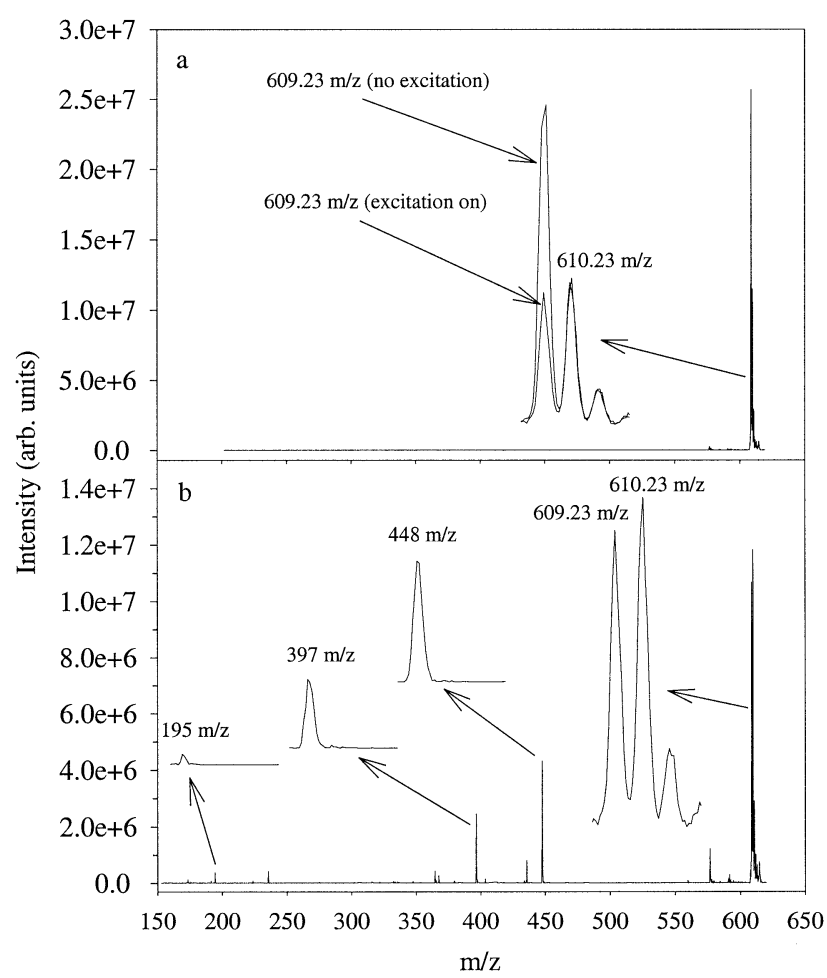

Figure 2. Mass spectra of reserpine: (a) No excitation, (b) fragmented by an auxiliary signal of frequency $60.380 \mathrm{kHz}$ and amplitude $50 \mathrm{mV}$ applied for $100 \mathrm{~ms}$. The inset in the upper frame shows the non-excited and excited precursor isotopic distributions overlapped using the same scale.

$$
\frac{d^{2} \vec{r}}{d t^{2}}=\frac{e}{m} \vec{E}
$$

where $\vec{r}$ is the position vector of the ion, $e$ is the electronic charge, $m$ is the mass of the ion, and $\overrightarrow{\mathrm{E}}$ is the electric field. Eq 4 was integrated numerically using Richardson extrapolation and the Bulirsch-Stoer method with adaptive step-size and error control [18]. With the exception of the pure hyperbolic case, for which an analytic expression exists, the electric field was obtained by interpolating tabulated numerical solutions to the Laplace equation. The numerical solutions were obtained using a relaxation solver developed in-house.

\section{Results and Discussion}

\section{Resonance Excitation versus High Energy CAD}

Figure 2 shows the fragment-ion spectrum of reserpine, $\mathrm{m} / \mathrm{z} 609.23$, over the mass range 150 to $620 \mathrm{Da}$. The isotopic cluster of reserpine was selected in the first analyzing quadrupole (Q1), transmitted through the Q2 collision cell at low energy to avoid fragmentation, and accumulated in Q3, from which ions were scanned via MSAE at $1000 \mathrm{Da} / \mathrm{s}$.

The upper frame in Figure 2 shows a spectrum obtained in the absence of resonant excitation in Q3. The lower frame shows the fragment-ion spectrum, which obtained after a dipolar auxiliary signal of am- 


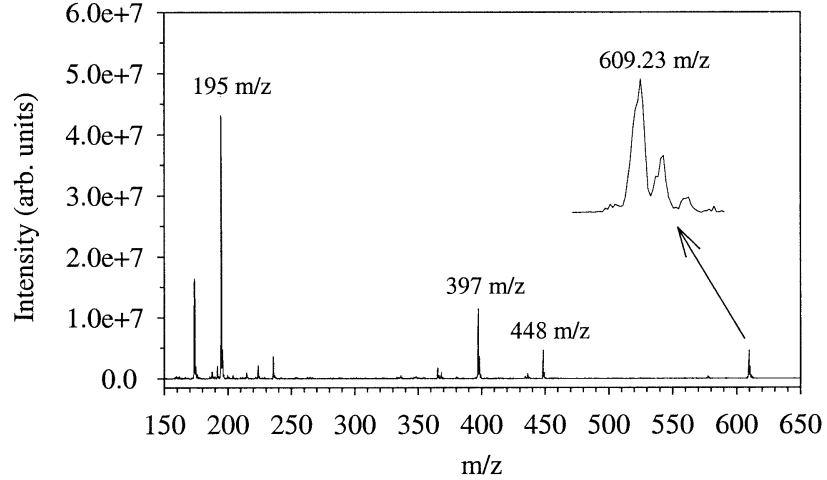

Figure 3. Product ion spectrum of reserpine injected into the Q2 collision cell $\left(5 \mathrm{mTorr}^{\mathrm{N}}\right)$ at $37 \mathrm{eV}$.

plitude $50 \mathrm{mV}$ and frequency $60.380 \mathrm{kHz}$ was applied for $100 \mathrm{~ms}$. In this case, the excitation frequency was tuned to maximize fragmentation of the 609.23 isotope. The insets in each frame show the isotopic distributions of the parent ion and selected fragment ions. The precursor isotopic distribution in Figure $2 \mathrm{~b}$ has been overlaid upon the precursor isotopic distribution of Figure 2a. Notice that the 609.23 isotope undergoes significant depletion while no depletion of 610.23 ion has occurred. Integrating the area under the 609.23 isotopic peak and the associated fragment peaks indicates that $41 \%$ of the 609.23 ion was converted into fragments and $21 \%$ was neutralized on the rods.

Shown in Figure 3 for comparison, is a fragment-ion spectrum obtained by selecting the parent ion isotopic cluster in Q1 and injecting it into the Q2 collision cell, containing 5 mTorr $\mathrm{N}_{2}$, at $37 \mathrm{eV}$. At this collision energy, the distribution of fragment ions is biased towards low mass when compared to the fragment-ion spectrum of Figure 2. The fragmentation efficiency is greater than $85 \%$ for this spectrum. This comparison illustrates that significantly more energy is available for fragmentation when the parent ion is injected into the Q2 collision cell than when resonant excitation is applied in Q3. In addition, collision-cell fragmentation is non-selective: all isotopes undergo CAD simultaneously. This is shown by the inset in Figure 3 where all of the isotopes of the parent ion have decreased in intensity.

\section{Auxiliary Frequency}

Figure 4 shows the frequency response of the 609.23 and 610.23 isotopes to an auxiliary dipolar signal of amplitude $50 \mathrm{mV}$, applied for $100 \mathrm{~ms}$. Included is the integral of the fragment-ion spectra over the range 150 to 600 Da. The depletion curves for both 609.23 and 610.23 exhibit full widths at half minimum (FWHM) of about $50 \mathrm{~Hz}$, yielding a resolution of excitation of about 60,000 $\mathrm{Hz} / 50 \mathrm{~Hz}=1200$. Resolution of excitation is defined as the mass of the subject ion divided by the half-width of its frequency response curve, transposed to the mass domain. At low $q$, dividing the auxiliary frequency by the half-width of the frequency response curve can be

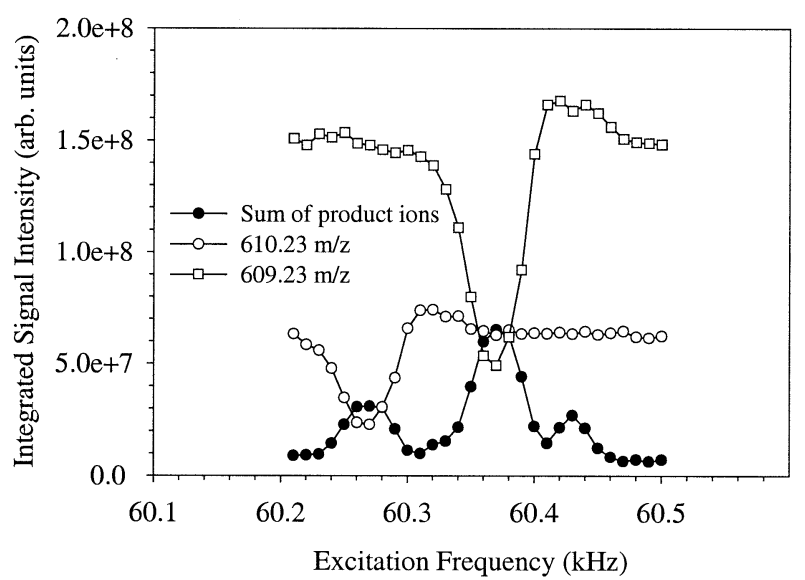

Figure 4. Frequency response of the 609.23 and 610.23 isotopes of reserpine to an auxiliary signal of amplitude $50 \mathrm{mV}$ applied for $100 \mathrm{~ms}$.

used to approximate this prescription. Clearly, a resolution of 1200 is adequate to resolve the 609.23 and 610.23 isotopes, which are separated in frequency-space by only $100 \mathrm{~Hz}$.

\section{Auxiliary Amplitude}

Figure 5 shows the integrated intensities of reserpine parent and fragment ions as a function of the amplitude of an auxiliary signal applied at $60.380 \mathrm{kHz}$ for $100 \mathrm{~ms}$. This data was collected with the Q1 transmitting quadrupole set at unit resolution to transmit only the 609.23 isotope into the LIT. Unit resolution was used because of the high selectivity of the excitation process which excites only one isotope at lower excitation amplitudes. Notice that the intensity of the fragment ions reaches a maximum near $90 \mathrm{mV}$ and then decreases steadily with increasing amplitude. The degree of fragmentation, at this excitation amplitude, is estimated to be greater than $90 \%$. In addition, the total intensity, including both parent and fragments, begins to decrease beyond am-

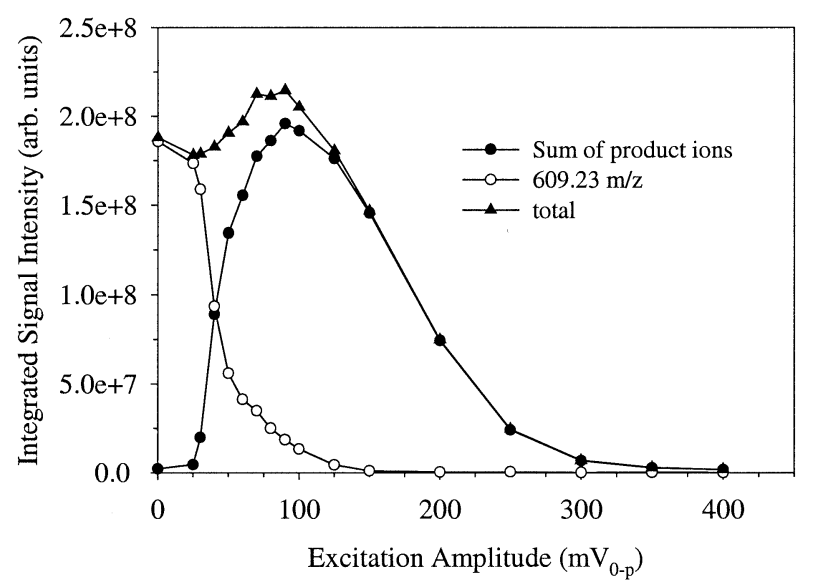

Figure 5. Intensity of the 609.23 isotope of reserpine and associated fragment-ions as a function of the amplitude of an auxiliary signal applied for $100 \mathrm{~ms}$ at $60.380 \mathrm{kHz}$. 


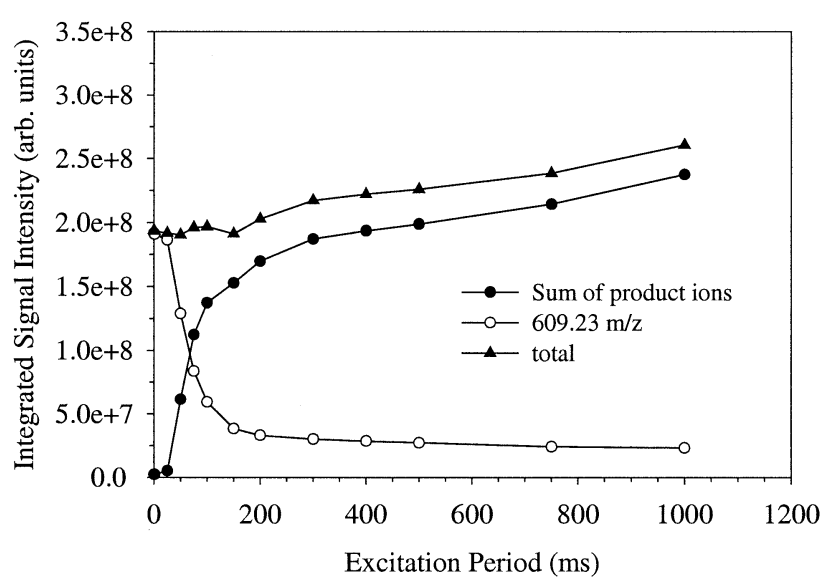

Figure 6. Intensity of the 609.23 isotope of reserpine and associated fragment-ions as a function of exposure to an auxiliary signal of frequency $60.380 \mathrm{kHz}$ and amplitude $50 \mathrm{mV}$.

plitude $90 \mathrm{mV}$ and decreases steadily thereafter: Evidence that the onset of ejection occurs at a low amplitude.

\section{Duration of Exposure}

The effects of varying the excitation period were examined and the results are shown in Figure 6. In this experiment, the auxiliary amplitude and frequency were held constant at $50 \mathrm{mV}$ and $60.380 \mathrm{kHz}$ respectively. Under these conditions the onset of fragmentation occurred after an exposure of about $25 \mathrm{~ms}$ with the degree of fragmentation increasing rapidly with exposure to near the $200 \mathrm{~ms}$ mark; after that, the increase was more gradual. When the total charge remains constant, the explanation for this behavior is not obvious. However, one aspect of the decrease in precursor ion intensity can be understood by considering that the bandwidth of the auxiliary signal (the inverse of its duration) diminishes with exposure [19]. Each collision disrupts the phase relationship between an ion's secular motion and the auxiliary signal. As the bandwidth of the auxiliary signal narrows with increasing duration, the probability of adequate phase continuity, for continued excitation following a collision, is reduced. In consequence of a collision-induced phase disruption, a narrow-band auxiliary signal is as likely to dampen an ion's secular motion as continue to excite. Another aspect which may contribute to the gradual increase in total ion intensity of Figure 6 is a slightly higher detection efficiency for low mass ions. The parameters affecting the relative detection efficiency of ions of differing masses is the subject of further detailed investigations.

\section{Unimolecular Dissociation}

Another mechanism which can lead to a continuing decrease in the intensity of the 609.23 isotope is the rate of unimolecular dissociation. In this work, the dissoci-

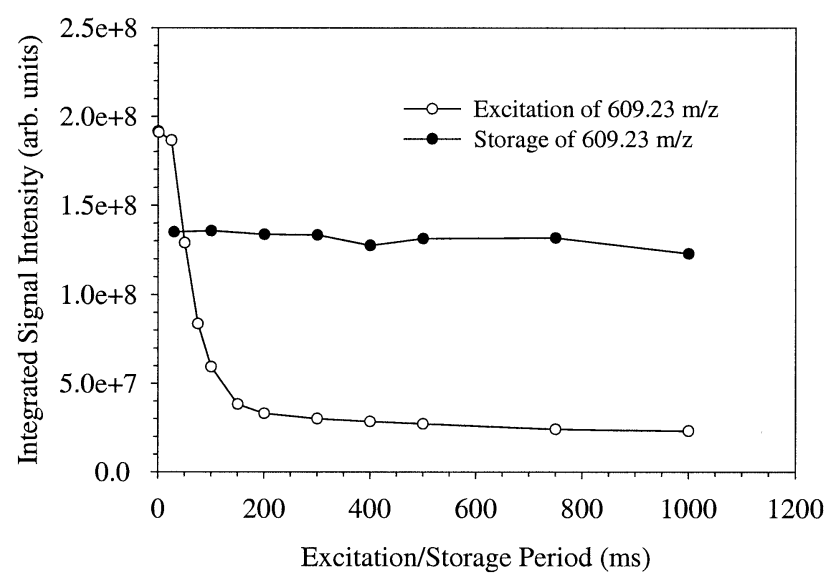

Figure 7. Intensity of the 609.23 isotope of reserpine as a function of excitation period (open circles) and storage period (solid circles). The storage period was the duration of the pause following a $100 \mathrm{~ms}$ exposure to resonant excitation before scanning out. In both cases the auxiliary signal was applied at a frequency of $60.380 \mathrm{kHz}$ and an amplitude of $50 \mathrm{mV}$.

ation mechanism was assumed to be fast on the time scale of the excitation and this is confirmed by the data of Figure 7. Represented by open circles is data for the 609.23 isotope, from Figure 6, where intensity is plotted as a function of exposure. The second set of data in Figure 7, solid circles, shows the intensity of the 609.23 isotope, excited resonantly for $100 \mathrm{~ms}$, and then stored for periods ranging from 30 to $1000 \mathrm{~ms}$. Note that the $x$-axis represents two different parameters: Exposure for the first data set and storage time for the second. Clearly, the storage period has no effect on the intensity of the 609.23 ion, confirming that 609.23 dissociates rapidly on the time scale of these experiments and that the decrease in 609.23 with increased exposure is due to resonant excitation and not unimolecular dissociation.

\section{The Consequences of Higher Order Fields in the Trapping Potential}

Based on the theory of forced damped harmonic oscillations, it was expected that exciting an ion for extended periods of time would lead to substantial losses on the rods. However, in the low-pressure environment of the LIT, this theory has limited application for two reasons. The first and most obvious is the discrete nature of collisions. The ion-trajectory simulations, detailed in the next section, indicate that the collision frequency for reserpine with thermal $\mathrm{N}_{2}$ is about $3.6 \mathrm{kHz}$ during the excitation process, one-seventeenth the secular frequency at $q=0.2$. In the FDHO model, damping is a continuous function, not representative of collisions in the LIT, where the phase discontinuities caused by discrete, infrequent collisions play a significant role.

A second limitation relates to the form of the radial trapping potential. Implicit in its name, the FDHO model assumes a harmonic potential for which the restoring force is linear with displacement. However, 
the round-rod geometry of the LIT introduces higher order terms, whose contribution to the potential increases with radial displacement $[20,21]$. In consequence of these higher order terms, the frequency of an ion's motion varies with its radial displacement [22, 23]. Accordingly, an ion can go off-resonance at higher radial amplitudes, to the extent that its radial motion is damped rather than excited. The result is alternating cycles of excitation and damping as the frequency of the ion's secular motion goes in and out of resonance with the auxiliary signal. This behavior is contrary to FDHO theory wherein secular frequency is independent of amplitude. These effects are illustrated clearly by iontrajectory simulations in pure-quadrupolar and roundrod-generated fields (vide infra, Figures 10 and 11).

\section{Ion-Trajectory Simulations}

Ion-trajectory simulations were used to study the response of ions to an auxiliary dipolar signal, in general, and, in particular, to observe differences in the behavior of ions in pure-quadrupolar and round-rod-generated fields. In the simulations described below, the minimum distance between opposing rods, whether hyperbolic or round, was $2 r_{0}$, with $r_{0}=4.170 \mathrm{~mm}$. The radius of round rods was $4.695 \mathrm{~mm}$, yielding an $r / r_{0}$ ratio of 1.126. The drive frequency was $1.0 \mathrm{MHz}$ and the poleto-ground amplitude of the RF drive was $225.3 \mathrm{~V}$ corresponding, in a pure quadrupole field, to $q=0.2077$ and secular frequency $74.07 \mathrm{kHz}$. In all cases, the mass of the singly charged ion was 609.23 Da. Although the drive frequency, and hence the nominal secular frequency, of these simulations does not match the experimental value of $816 \mathrm{kHz}$, the principles elucidated are unaffected by this discrepancy.

\section{Computer Simulations of Resonant Excitation}

Simulations were used to measure the frequency response of $\mathrm{m} / \mathrm{z} 609.23$ during a $50 \mathrm{~ms}$ exposure to a dipolar auxiliary signal of amplitude $25 \mathrm{mV}$, in a round-rod-generated field, in the presence of 0.03 mTorr $\mathrm{N}_{2}$. The duration of the exposure, number of collisions and the total energy loss experienced by each of 100 ions were averaged for specific auxiliary frequencies over a range of frequencies between 73.3 and 75.1 $\mathrm{kHz}$. These data, presented in Figure 8, show that response was strongest at $74.225 \mathrm{kHz}$. This value differs little from the theoretical prediction for a pure quadrupole field of $74.067 \mathrm{kHz}$. Near the resonant frequency, some ions were lost on the rods before the $50 \mathrm{~ms}$ exposure was complete. Under normal experimental conditions, it is probable that these ions would have fragmented before being lost.

The total energy loss plotted as a function of frequency in Figure 8 is the sum of the kinetic energy that an ion loses during each collision event over the course of the trajectory. In some instances, when their precollision kinetic energy is relatively low, ions can gain

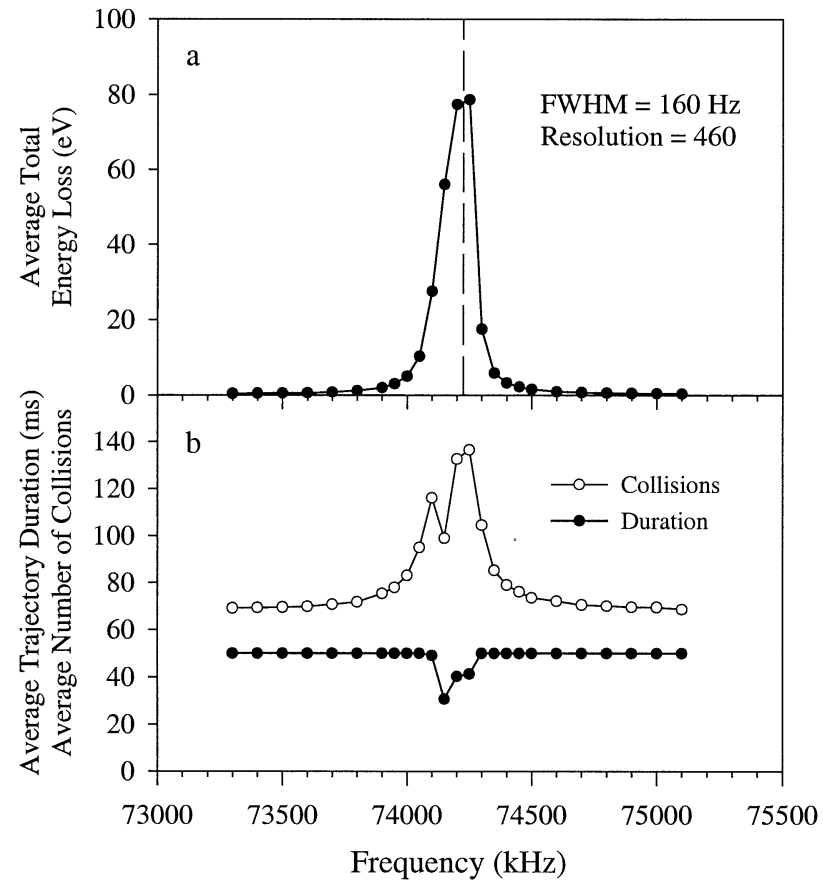

Figure 8. Simulated frequency response of mass 609.23 at $q=$ $0.2077(\Omega / 2 \pi=1.0 \mathrm{MHz})$ to an auxiliary signal of amplitude 25 $\mathrm{mV}$ for $50 \mathrm{~ms}$ in $0.03 \mathrm{mTorr}_{2}$ : (a) Average total energy loss and (b) trajectory duration (solid circles) and number of collisions (open circles).

kinetic energy from a neutral, resulting in negative contributions to total energy loss. Even so, more frequently the loss is positive and the energy available for transfer from kinetic to internal energy of the ion is equal to the centre of mass collision energy-approximately half of the total energy loss. When ions were on or near resonance, the average energy loss (total energy loss/number of collisions) was $0.57 \mathrm{eV}$, compared to $0.0023 \mathrm{eV}$ when there was no excitation.

It was evident from the fragmentation observed experimentally that the energy stored internally in the ion had sufficient time to accumulate and cause dissociation. This is an indication that the dissipation rate of internal energy through thermalizing collisions and radiative cooling was low compared to the dissociation rate. This was not the case for low-amplitude excitation of ions at higher pressures where high rates of thermalization resulted in significant thresholds for auxiliary amplitude, below which fragmentation was not observed [3].

An interesting feature of Figure 8 is the increase in the number of collisions when the auxiliary signal was on, or near, resonance. In the absence of excitation, the average number of collisions was 68, compared to 135 for the on-resonance condition. This increase was a consequence of the increased path length of higheramplitude ions. For example, the total path length of the ion trajectory shown in Figure 9 was 50.38 m, while that of an unexcited thermal trajectory was only $7.17 \mathrm{~m}$.

The disproportionate increase in path length, a factor 


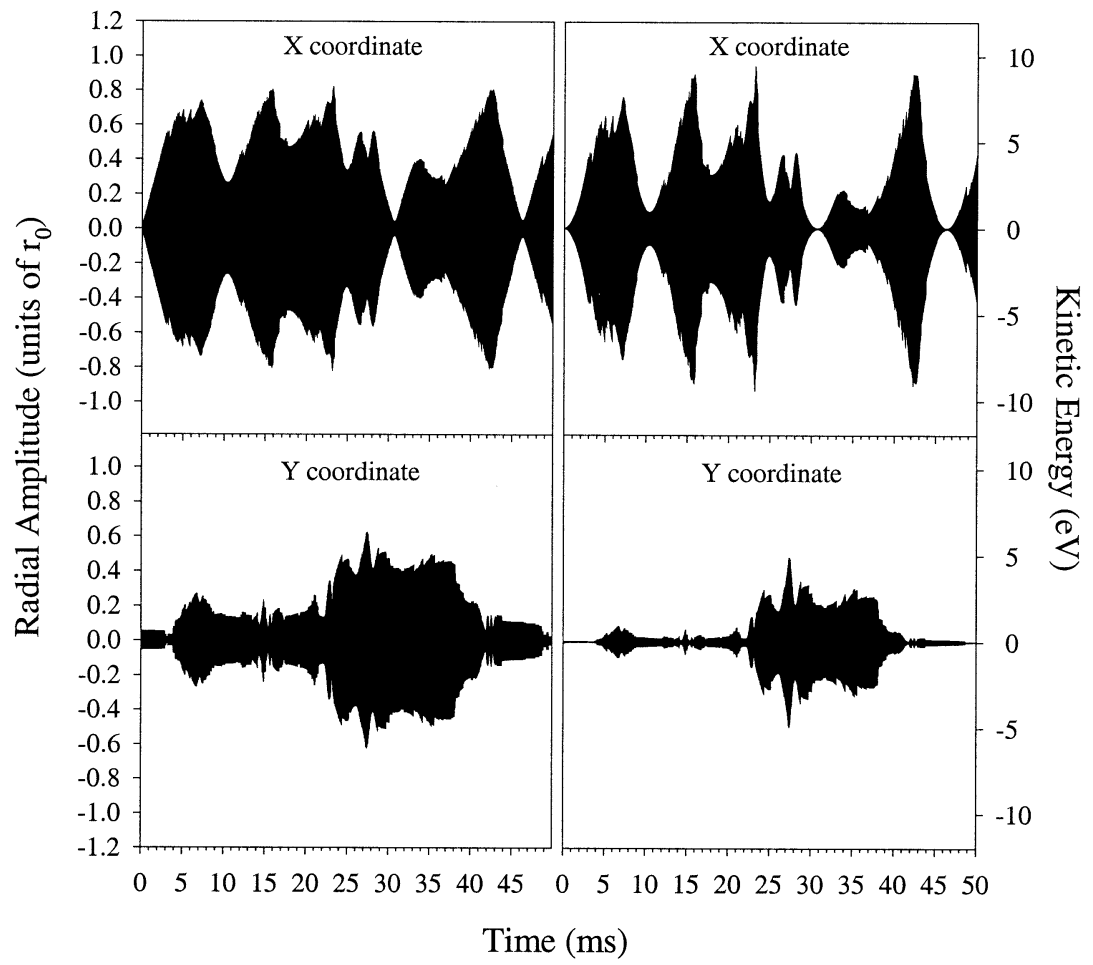

Figure 9. The displacement and kinetic energy of mass 609.23 in the $x$ and $y$ directions as a function of time. The ion was excited by an auxiliary signal of frequency $74.225 \mathrm{kHz}$ and amplitude $25 \mathrm{mV}$ in $0.03 \mathrm{mTorr} \mathrm{N}_{2}$. Electric potentials were applied to round rods.

of 7, compared to the increase in number of collisions, a factor of 2 , is a result of the inclusion of the thermal motion of the collision gas. For example, if the collision gas was assumed to be stationary, the number of collisions suffered by the ion would be dependent solely upon the collision cross section times the path length that the ion sweeps out during a period of time, $\mathrm{T}$. If the ion has zero kinetic energy, the path length would equal zero and the number of collisions in time period $\mathrm{T}$ would also be equal to zero. However, the inclusion of the thermal energy for the collision gas ensures that the ion undergoes a minimum number of collisions during the time period $\mathrm{T}$ even if the ion were stationary. The number of collisions is then proportional to the relative velocity of the ion and neutral where the magnitude of the relative velocity is defined as [24]

$$
V_{r e l}^{2}=V^{2}+U^{2}-2 V U \cos \theta
$$

where $V$ is the thermal velocity of the collision gas, $U$ is the velocity of the ion and $\theta$ is the centre of mass scattering angle. The average centre of mass scattering angle can be taken as $90^{\circ}$ [25]. At low ion kinetic energy the thermal motion of the gas becomes significant whereas at high kinetic energies $(>1-2 \mathrm{eV}$ in the case of the ion reserpine and nitrogen as the collision gas) the gas thermal velocity becomes insignificant.

The ion trajectories shown in Figures 9 and 10 were obtained using auxiliary amplitude $25 \mathrm{mV}$, applied at the resonant frequency of $74.225 \mathrm{kHz}$, with $\mathrm{N}_{2}$ pressures of 0.03 and 0 mTorr, respectively. Note that the radial components of the ion's velocity are expressed in units of eV. In Figure 10, the trajectory displays a complicated beat pattern in both the $x$ and $y$ directions even though the dipolar auxiliary signal was applied between the $x$ poles only. This behavior is a consequence of the round-rod geometry, which superposes with the quadrupole potential higher-order terms whose significance increases with radial amplitude. The origin of the beat pattern can be distinguished from a beat pattern arising from exciting off-resonance by the existence of the growth in the ion trajectory amplitude in the $y$-direction while exciting in the $x$-direction. This arises from the existence of higher order terms in the trapping potential. Off-resonance excitation in the $x$-direction does not lead to growth in the y-direction. Comparison of Figures 9 and 10 shows that neither radial amplitude nor radial energy was reduced significantly by the addition of 0.03 mTorr $N_{2}$. This is because the collision frequency was low relative to the resonant frequency. Specifically, the ion of Figure 9 suffered a total of only 178 collisions during the $50 \mathrm{~ms}$ exposure, corresponding to an average of 20.8 secular cycles between collisions. As a result of this relatively low collision frequency of $3.6 \mathrm{kHz}$, the ion accumulated radial kinetic energy of nearly $10 \mathrm{eV}$. Collisions suffered at this energy would impart significant internal energy to an ion.

Of significance in Figures 9 and 10 is the extent to which motion in the $y$-direction was excited by a 


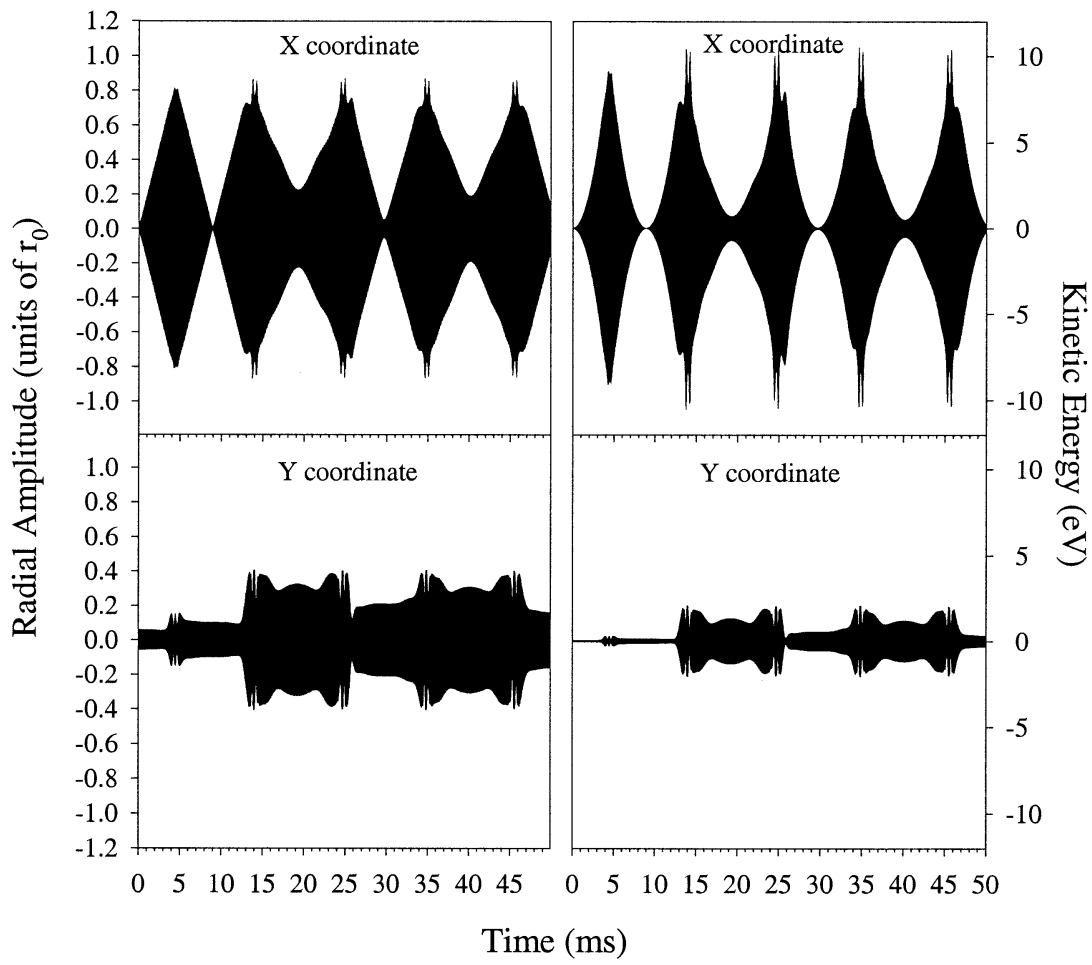

Figure 10. The displacement and kinetic energy of mass 609.23 in the $x$ and $y$ directions as a function of time. The ion was excited by an auxiliary signal of frequency $74.225 \mathrm{kHz}$ and amplitude $25 \mathrm{mV}$ in the absence of any buffer gas. Electric potentials were applied to round rods.

dipolar signal applied between the $x$-rods. As mentioned above, this is a consequence of higher terms in the drive potential introduced by round-rod electrodes.

A general expression for a two-dimensional multipole field can be written as

$$
\Phi(x, y)=\sum_{n=0}^{\infty} \phi_{\mathrm{n}}(x, y)=\sum_{\mathrm{n}=0}^{\infty} A_{\mathrm{n}} \operatorname{Re}\left(\frac{x+i y}{r_{0}}\right)^{\mathrm{n}}
$$

where $2 \mathrm{n}$ is the number of poles in a particular term. For example, $\mathrm{A}_{2}$ is the amplitude of the quadrupole contribution. In the work presented here, both experimental and simulated, the $r / r_{0}$ ratio was 1.126 . In a previous publication [20], it was shown that the coefficients obtained from this ratio are $\mathrm{A}_{2}=1.001462, \mathrm{~A}_{6}=$ $0.001292, \mathrm{~A}_{10}=-0.002431$ and $\mathrm{A}_{14}=-0.0002975$ with all other terms for which $n<14$ equal to zero. The resulting electric potential is given by

$$
\begin{aligned}
\Phi_{r / r_{0}=1.126}= & A_{2} \frac{x^{2}-y^{2}}{r_{0}^{2}} \\
& +A_{6} \frac{x^{6}-15 x^{4} y^{2}+15 x^{2} y^{4}-y^{6}}{r_{0}^{6}}+ \\
& +A_{10} \frac{-210 x^{6} y^{4}+45 x^{2} y^{8}-y^{6} y^{4}}{r_{0}^{10}} \\
& +\ldots
\end{aligned}
$$

Several features of eq 7 are relevant to this discussion. Only in the case of a pure quadrupole potential, for which the restoring force is both proportional to the displacement and decoupled in the two coordinate directions, is ion-motion harmonic. Furthermore, the significance of the higher order terms, and therefore the strength of the $x-y$ coupling, increases dramatically with radial displacement. As a result, the frequency of an ion's secular motion in one coordinate direction becomes a function of amplitude in both coordinate directions. As demonstrated in Figure 10, the strength of this effect is sufficient for ions to go out of phase with the auxiliary signal at high radial amplitude resulting in alternating periods of resonant excitation and resonant de-excitation.

Figure 11 shows the trajectory of an ion moving in response to a pure quadrupolar RF potential, $q=$ 0.2077 , and a $25 \mathrm{mV}$ dipolar auxiliary signal applied between the $x$-rods at the theoretical secular frequency of $74.067 \mathrm{kHz}$. In this instance, the secular frequency was calculated using the continued fraction expression for $\beta$ [15]. The left and right frames correspond to nitrogen pressures of zero and 0.03 mTorr, respectively. In both cases, the ion was lost on a rod within $6 \mathrm{~ms}$. The low-pressure buffer gas resulted in 12 collisions, which impacted the trajectory minimally, extending the duration by only $0.8 \mathrm{~ms}$. The effects of the collisions are visible as slight discontinuities in the envelopes of both coordinate components of the trajectory. Furthermore, in the absence of collisions, there was no coupling of 


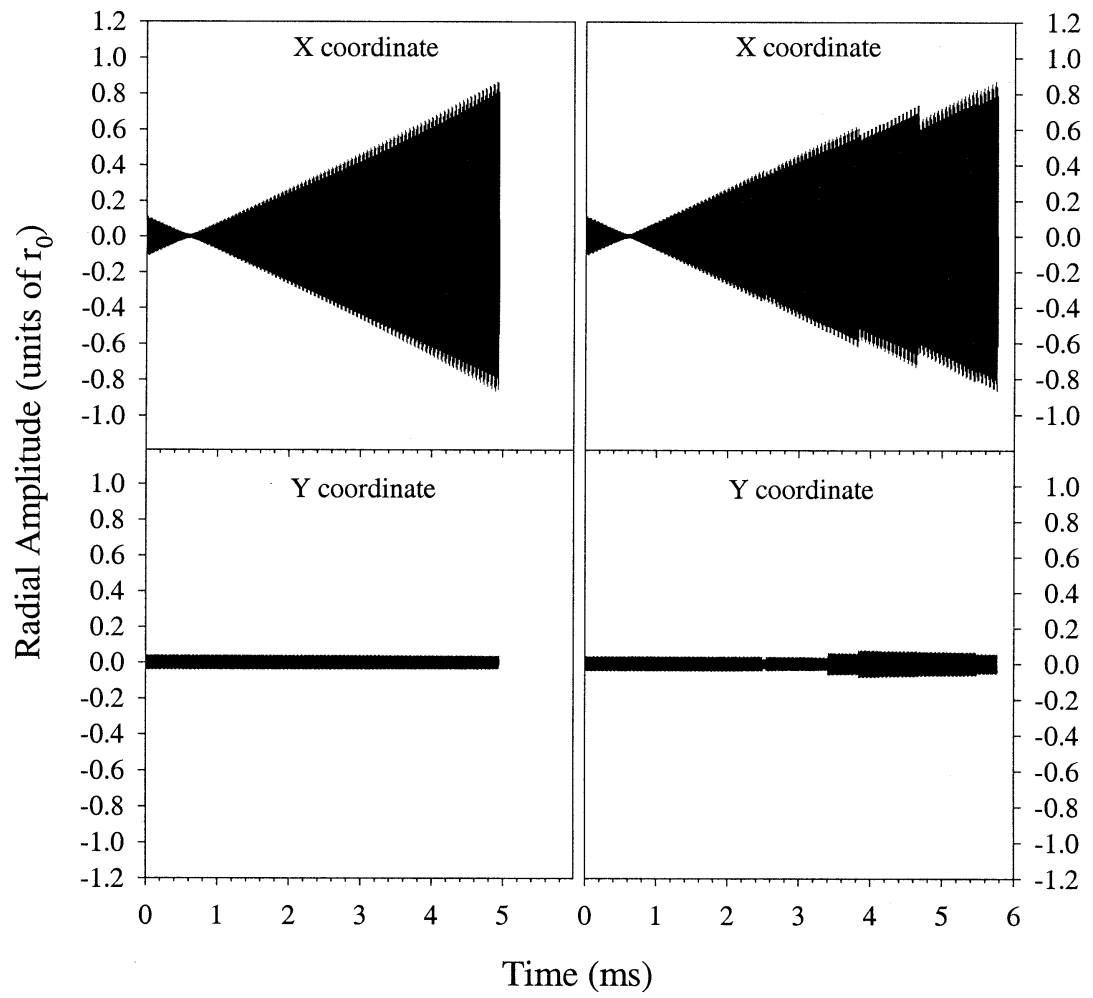

Figure 11. The displacement and kinetic energy of mass 609.23 in the $x$ and $y$ directions as a function of time. The ion was excited by an auxiliary signal of frequency $74.067 \mathrm{kHz}$ and amplitude $25 \mathrm{mV}$. The left and right frames correspond to $\mathrm{N}_{2}$ pressures of 0 and $0.03 \mathrm{mTorr}$, respectively. The RF potential was applied to hyperbolic rods to obtain a pure quadrupolar field.

motion in the two coordinate directions and the ion remained on-resonance with the auxiliary signal until it was neutralized on the rods.

Figures 8 to 11 demonstrate clearly the advantages of round-rod generated fields over pure quadrupole geometries for the purpose of ion-fragmentation via resonant excitation at low pressures. In the absence of the higher order terms in eq 7, only the most labile ions would be confined sufficiently long to fragment before being lost on the rods.

\section{Fragmentation of Higher Mass Ions}

Consideration was given to fragmentation of a higher mass ion. The ion chosen for this study was a homogeneously substituted triazatriphosphorine of mass of 2721.89 Da [26]. Larger ions contain significantly more internal degrees of freedom than smaller ions and consequently must gain more internal energy to dissociate. Additional energy can be made available to an ion by increasing either the auxiliary amplitude or the excitation period. Figure 12 shows mass spectra of the 2722 ion before and after resonant excitation by a dipolar auxiliary signal amplitude of $400 \mathrm{mV}$ applied for $200 \mathrm{~ms}$. Insets show the isotopic distributions of the parent and its 2290 Da fragment. The lower frame shows the fragmentation of the 2722 isotope at a frequency of $59.800 \mathrm{kHz}$ with $q=0.207$. Under these

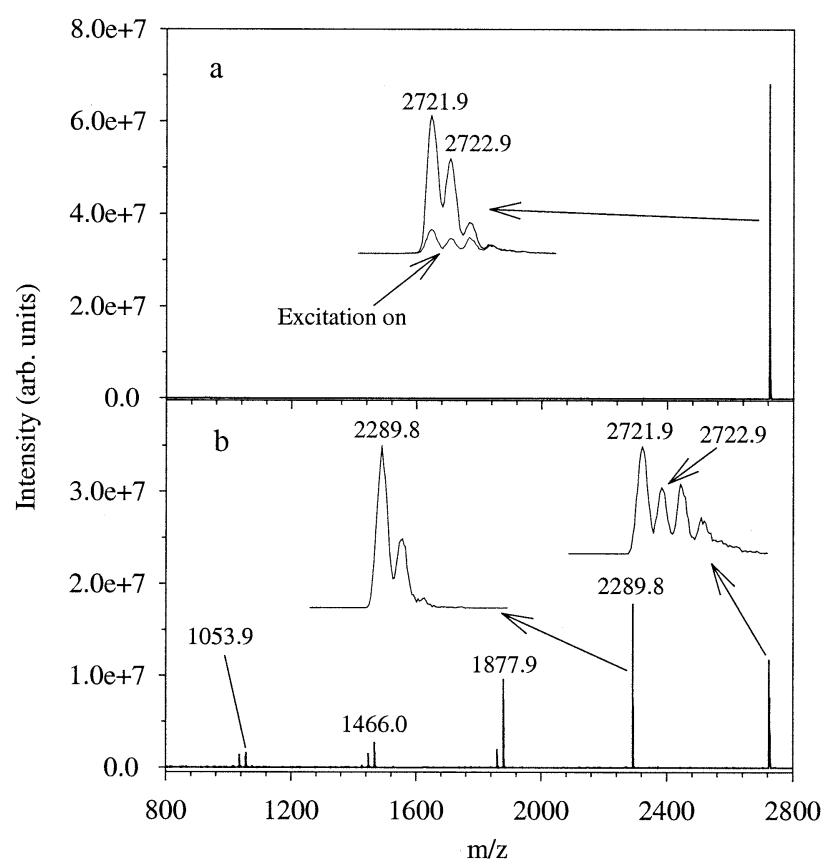

Figure 12. The upper frame (a) shows the mass spectrum of a homogeneously substituted triazatriphosphorine $(\mathrm{m} / \mathrm{z}$ 2721.89) with no excitation applied. The lower frame $(\mathbf{b})$ shows the fragmentation pattern that results when a $400 \mathrm{mV}$ auxiliary signal is applied at $59.800 \mathrm{kHz}$ for a period of $200 \mathrm{~ms}$. The insets show the isotopic patterns of some selected peaks. The pressure in the LIT was $0.045 \mathrm{mTorr} \mathrm{N}_{2}$. The inset in the upper frame shows the non-excited and excited precursor isotopic distributions overlapped using the same scale. 


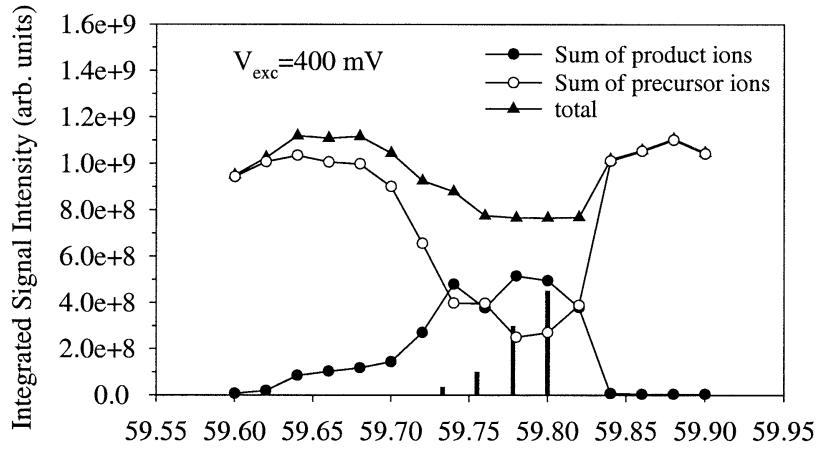

Auxiliary Frequency $(\mathrm{kHz})$

Figure 13. Abundance of the 2721.89 isotopic cluster and its associated fragment-ions in response to a $200 \mathrm{~ms}$ exposure to an auxiliary signal of amplitude $400 \mathrm{mV}$. The vertical bars indicate the experimentally measured intensities of the isotopes at their theoretically predicted secular frequencies. Note that the highest frequency corresponds to the lightest ion.

conditions the integrated area of the fragments indicates that $48 \%$ of the $\mathrm{m} / \mathrm{z} 2722$ isotopic cluster was fragmented in the excitation process, while $25 \%$ was neutralized on the rods.

Figure 13 shows the frequency response of the isotopic cluster to an auxiliary signal of amplitude $400 \mathrm{mV}$ applied for $200 \mathrm{~ms}$. Under these conditions, the responses of individual isotopes were not resolved. The response curve shows a sharp edge on the high frequency side (low mass), which then tails off towards lower frequency (high mass). The secular frequencies predicted by eq 3 of the first four isotopes, 2722 to 2725 , are shown as vertical bars in the lower frame. The value of $q$ was calculated for each isotope using

$$
q=\frac{4 e V_{R F}}{m r_{0}^{2} \Omega^{2}}
$$

where $V_{R F}$ is the pole to ground RF amplitude, $r_{0}$ is the field radius, $\Omega$ is the drive frequency and $\mathrm{m}$ is the mass of the ion. During the excitation process $V_{R F}$ is constant for all ions within the LIT. This results in a frequency spacing of $\approx 22 \mathrm{~Hz}$ between neighbouring isotopes. The heights of the vertical bars correspond to the experimental intensities of the unexcited isotopic cluster.

It should be noted that the frequency response curve appears to be distorted on the low frequency side. The response curves for the $2722 \mathrm{Da}$ parent and its $2290 \mathrm{Da}$ fragment, Figure 14, exhibit asymmetries similar to the response curves for the entire isotropic cluster, Figure 13 , demonstrating that asymmetry is a consequence of the excitation process and not a convolution of isotopic contributions. An explanation of the factors which determine the shape of frequency-response curves requires further investigation and falls outside the scope of this paper, although it should be noted that inaccuracies in the rod alignment [27], the existence of nonuniformities in the surface potential of the rods [22], and as well the presence of the higher order fields [28]

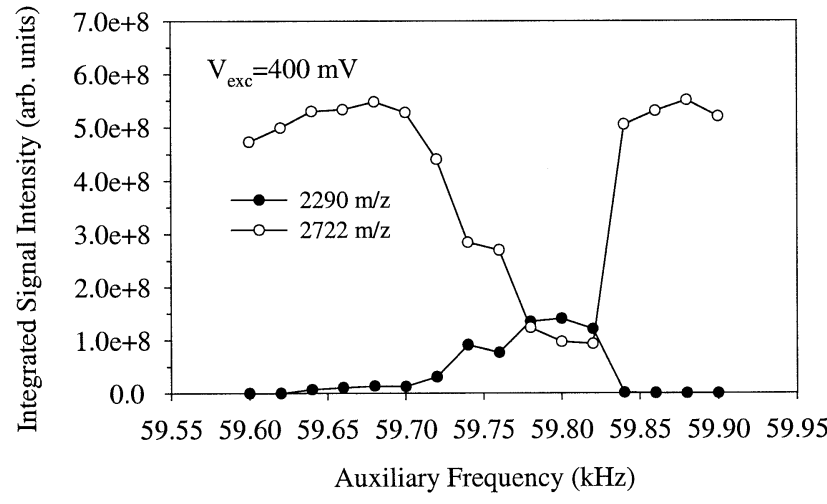

Figure 14. Abundance of the 2721.89 isotope and its $2290 \mathrm{Da}$ fragment in response to a $200 \mathrm{~ms}$ exposure to an auxiliary signal of amplitude $400 \mathrm{mV}$.

could lead to potential distortions of the frequencyresponse curves.

The degree of excitation is a function of the amplitude and duration of the auxiliary signal. Figure 15 shows the effects of varying the auxiliary amplitude for exposures of 100 and $200 \mathrm{~ms}$. In both cases, losses on the rods were similar. Even so, increasing the exposure from 100 to $200 \mathrm{~ms}$ led to an increase in the production of fragment ions with a concomitant decrease in the intensity of the parent ion cluster. However, the increase in exposure did not influence the proportion in which isotopes were excited (data not shown). By contrast, increasing the auxiliary amplitude broadens the frequency response, increasing the range of isotopes that respond at a specific frequency. This behavior is a

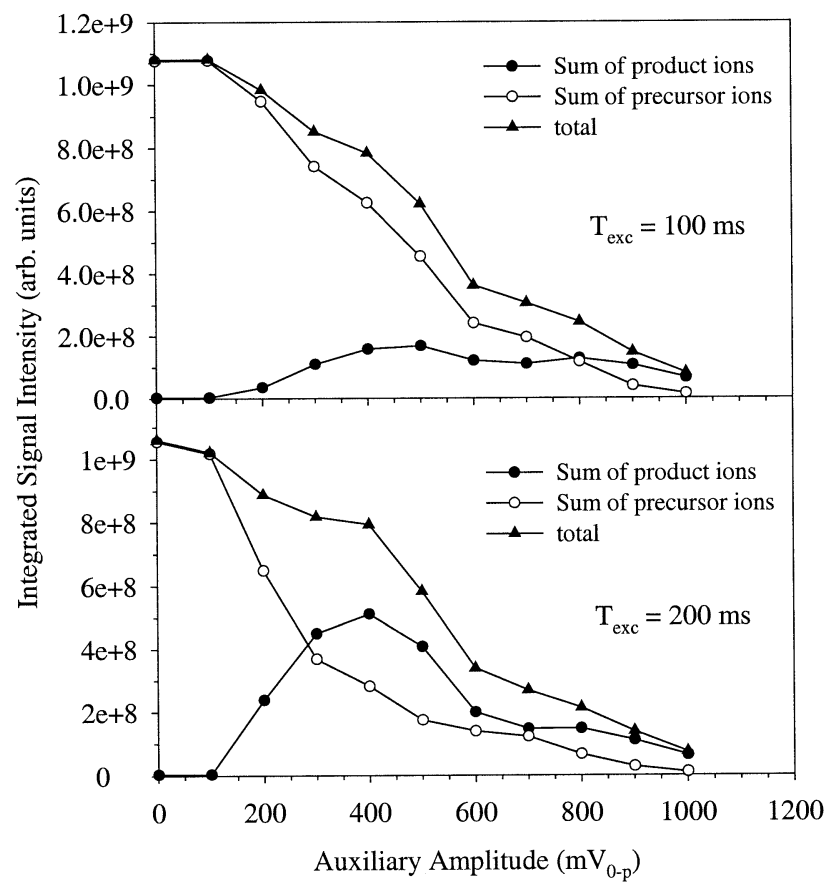

Figure 15. Abundance of the 2721.89 isotopic cluster and its associated fragment-ions as a function of the amplitude of a 59.800 $\mathrm{kHz}$ auxiliary signal for exposures of (a) $100 \mathrm{~ms}$ and (b) $200 \mathrm{~ms}$. 


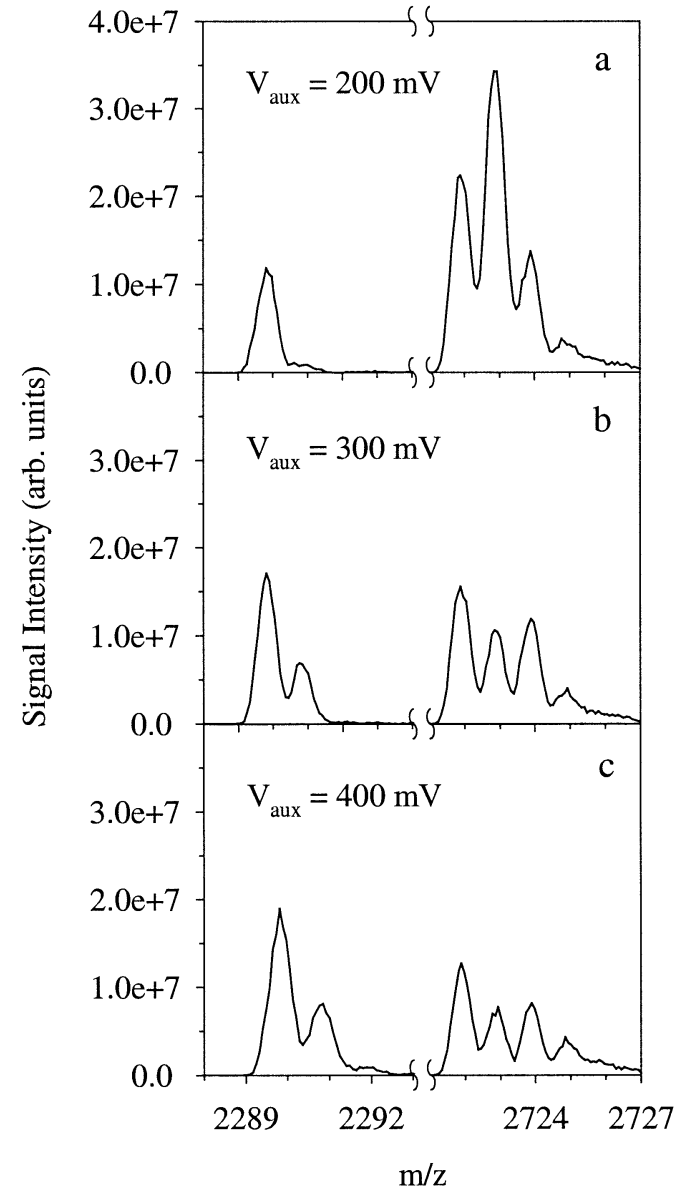

Figure 16. The isotopic distributions of the 2721.89 and 2290 clusters following $200 \mathrm{~ms}$ exposure to $59.800 \mathrm{kHz}$ auxiliary signal for amplitudes of (a) $200 \mathrm{mV}$, (b) $300 \mathrm{mV}$, and (c) $400 \mathrm{mV}$.

function of the bandwidth of the auxiliary waveform and the critical energy that must be absorbed by an isotope before fragmentation or neutralisation can occur [3]. The effect is illustrated by Figure 16, which shows the $2290 \mathrm{Da}$ fragment-ion cluster obtained with auxiliary amplitudes 200, 300, and $400 \mathrm{mV}$, each applied for 200 ms. Clearly, as the amplitude increased, fragmentation of the second (2723 Da) and third (2724 Da) isotopes led to the increased intensities of the second (2291 Da) and third (2292 Da) fragment isotopes.

Figure 17 displays the integrated intensities of fragment and parent ions as a function of exposure for auxiliary amplitudes 200 and $400 \mathrm{mV}$. In each case, the degree of fragmentation increased for a few hundred $\mathrm{ms}$ and then remained constant. At the higher amplitude of $400 \mathrm{mV}$, the plateau occurred at greater relative intensity and was attained more quickly. As noted previously, increased auxiliary amplitude led to increased losses on the rods. This is evident in the reduction in total integrated intensity at the higher amplitude. The plateau is a consequence of the complete depletion of responsive ions.

Comparison of the 2290 and 2722 isotopic clusters after exposures of 200 and $1000 \mathrm{~ms}$ to an auxiliary signal

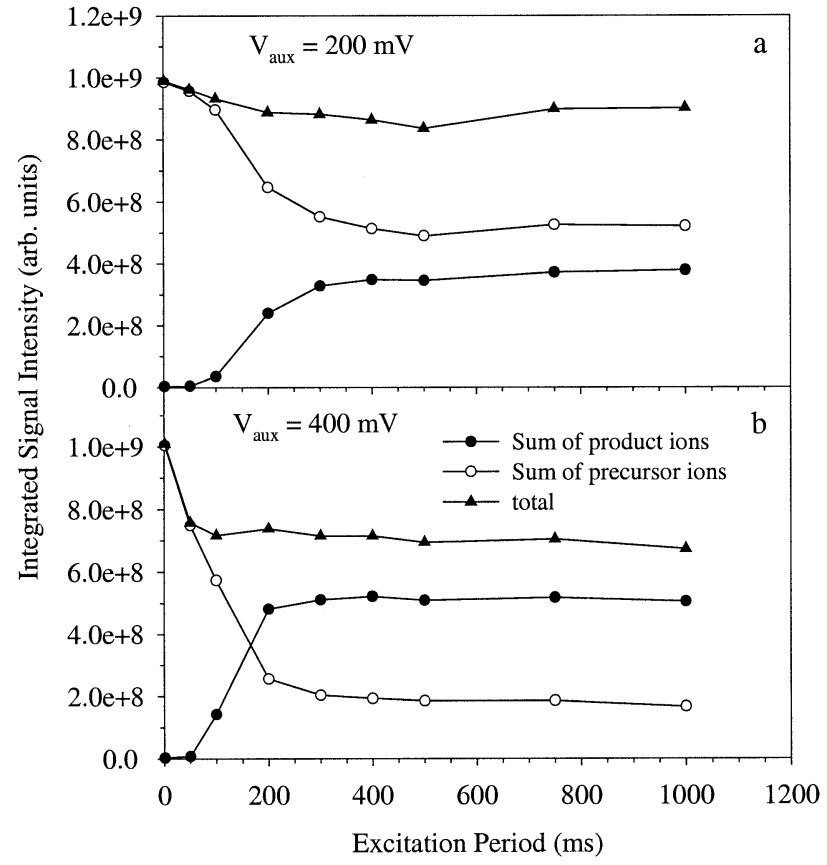

Figure 17. Abundance of the 2721.89 isotopic cluster and its associated fragment-ions as a function of exposure to an auxiliary signal of $59.800 \mathrm{kHz}$ of amplitude (a) $200 \mathrm{mV}$ and (b) $400 \mathrm{mV}$.

of amplitude $200 \mathrm{mV}$ is shown in Figure 18. These spectra reveal that the 2722 isotope was excited preferentially. Even so, increased excitation periods were unable to remove completely the 2722 Da isotope; $13 \%$ remained after $1000 \mathrm{~ms}$. This curiosity is speculated to arise from non-uniformities in the surface potential of

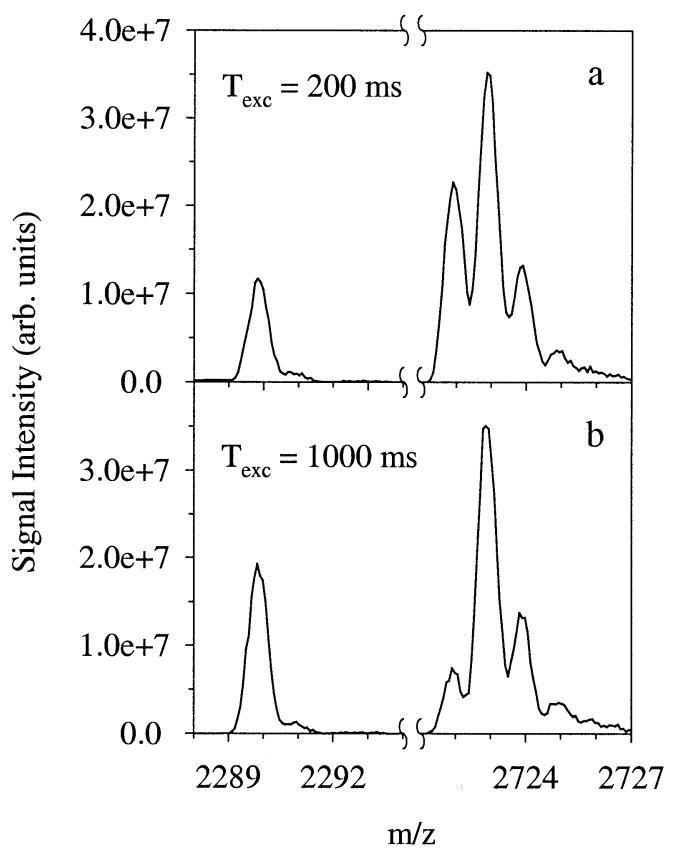

Figure 18. The isotopic distributions of the 2721.89 and 2290 clusters following irradiation by an auxiliary signal of frequency $59.800 \mathrm{kHz}$ and amplitude $200 \mathrm{mV}$ for exposures of (a) $200 \mathrm{~ms}$ and (b) $1000 \mathrm{~ms}$. 
the rods [22]. It is suggested that micro-potential wells could localize groups of ions in regions where the effective RF level was sufficiently different that their secular frequency would be off-resonance with the auxiliary signal. For example, a shift of $50 \mathrm{~Hz}$ in secular frequency at $q \approx 0.21$ would require a shift in RF level of about $0.54 \mathrm{~V}$ for $m / z 2722$, but only $0.12 \mathrm{~V}$ for $m / z 609$. Following the same reasoning, micro-potentials would cause broadening of frequency response data.

\section{Resolution of Resonant Excitation}

It was noted above that the strongest response to the auxiliary signal of the first and second isotopes of reserpine, $609.23 \mathrm{Da}$, occurred at frequencies separated by $100 \mathrm{~Hz}$. In contrast, it was calculated that only $22 \mathrm{~Hz}$ separated the frequencies of maximum response, of the first two isotopes of triazatriphosphorine, $2722 \mathrm{Da}$. This difference can be understood by considering the relationship between secular frequency and the stability parameter $q$, defined in eq 8 .

Combining eqs 3 and 8 gives the following expression for $\omega_{0}(\mathrm{~m})$,

$$
\omega_{0}(m) \approx \frac{\sqrt{2} e}{\Omega r_{0}^{2}} \frac{V_{R F}}{m}
$$

In these experiments, $V_{R F}$ was held constant at a value, such that $q \approx 0.21$ for the parent ion and the auxiliary frequency was varied to obtain the frequency response. Combining the constant terms on the r.h.s. of eq 9 into a single parameter, $C$, fundamental secular frequency is directly proportional to the RF level and inversely proportional to mass as

$$
\omega_{0}(m) \approx C \frac{V_{R F}}{m}
$$

Using eq 10, the difference in the fundamental secular frequency of two masses, $m_{1}$ and $m_{2}$, at RF amplitudes $V_{1}$ and $V_{2}$ can be written

$$
\Delta \omega=\omega_{0}\left(m_{1}\right)-\omega_{0}\left(m_{2}\right)=C\left(\frac{m_{2} V_{1}-m_{1} V_{2}}{m_{1} m_{2}}\right)
$$

In the case of the first and second isotopes of the ions used in this study, $V_{1}=V_{2}, m_{2}=m_{1}+1$, and $m_{2} \approx m_{1}=$ $m$, so eq 11 can be simplified to

$$
\Delta \omega \approx C \frac{V_{R F}}{m^{2}}
$$

Eq 12 predicts differences in the fundamental secular frequencies of the first and second isotopes of reserpine and triazatriphosphorine of 98.9 and $22.0 \mathrm{~Hz}$, respectively. The predicted value for reserpine is in good agreement with the experimental value of $100 \mathrm{~Hz}$.
More generally, while the relationship between secular frequency and the stability parameter $q$ remains fixed, the density of masses on the $q$-axis increases with $V_{R F}$. As a result, the separation between the fundamental secular frequencies of adjacent isotopes, and consequently the ability to excite individual isotopes exclusively, decreases with increasing mass. This condition can be appreciated quantitatively by differentiating eq 9 with respect to mass and using eq 8 to write $V_{R F}$ in terms of $q$ as

$$
\frac{\partial \omega_{0}}{\partial m}=-\frac{\Omega q}{2 \sqrt{2}} \frac{1}{m}
$$

Eq 13 shows that the change in fundamental secular frequency per unit mass decreases with increasing mass, but increases with the stability parameter $q$. Accordingly, the ability to excite individual isotopes exclusively increases with $q$.

\section{Conclusions}

Contrary to initial expectations, it has been shown that ions can be fragmented via resonant excitation with high efficiency and high resolution in a low-pressure LIT. To be effective, this technique requires extended excitation by a low amplitude auxiliary signal and higher order terms in the essentially quadrupolar trapping potential to reduce losses on the rods.

\section{References}

1. Campbell, J. M.; Collings, B. A.; Douglas, D. J. A New Linear Ion Trap Time-of-flight System with Tandem Mass Spectrometry Capabilities. Rapid Commum. Mass Spectrom. 1998, 12, 1463-1474.

2. Collings, B. A.; Douglas, D. J. Observation of Higher Order Quadrupole Excitation Frequencies in a Linear Ion Trap. J. Am. Soc. Mass Spectrom. 2000, 11, 1016-1022.

3. Collings, B. A.; Campbell, J. M.; Mao, D.; Douglas, D. J. A Combined Linear Ion Trap Time-of-flight System with Improved Performance and $\mathrm{MS}^{\mathrm{n}}$ Capabilities. Rapid Commum. Mass Spectrom. 2001, 15, 1777-1795.

4. Collings, B. A.; Sudakov, M.; Londry, F. A. Resonance Shifts in the Excitation of the $n=0, K=1$ to 6 Quadrupole Resonances for Ions Confined in a Linear Ion Trap. J. Am. Soc. Mass Spectrom. 2002, 13, 577-586.

5. Hart, K. J.; McLuckey, S. A. Relative Dissociation Energy Measurements Using Ion Trap Collisional Activation. J. Am. Soc. Mass Spectrom. 1994, 5, 250-259.

6. Charles, M. J.; McLuckey, S. A.; Glish, G. L. Competition Between Resonance Ejection and Ion Dissociation During Resonant Excitation in a Quadrupole Ion Trap. J. Am. Soc. Mass Spectrom. 1994, 5, 1031-1041.

7. Goeringer, D. E.; Whitten, W. B.; Ramsey, J. M.; McLuckey, S. A.; Glish, G. L. Theory of High-Resolution Mass Spectrometry Achieved via Resonance Ejection in the Quadrupole Ion Trap. Anal. Chem. 1992, 64, 1434-1439.

8. Goeringer, D. E.; McLuckey, S. A. Evolution of Ion Internal Energy during Collisional Excitation in the Paul Trap: A Stochastic Approach. J. Chem. Phys. 1996, 104, 2214-2221. 
9. Gauthier, J. W.; Trautman, T. R.; Jacobson, D. B. Sustained Off-Resonance Irraditation for Collision-Activated Dissociation Involving Fourier Transform Mass Spectrometry. Collision-Activated Dissociation Technique that Emulates Infrared Multiphoton Dissociation. Anal. Chim. Acta 1991, 246, 211-225.

10. Marshall, A. G.; Hendrickson, C. L.; Jackson, G. S. Fourier Transform Ion Cyclotron Resonance Mass Spectrometry: A Primer. Mass Spectrom. Rev. 1998, 17, 1-35 and references therein.

11. Mirgorodskaya, E.; O'Connor, P. B.; Costello, C. E. A General Method for Precalculation of Parameters for Sustained OffResonance Irradiation/Collision-Induced Dissociation. J. Am. Soc. Mass Spectrom. 2002, 13, 318-324.

12. Hager, J. W. A New Linear Ion Trap Mass Spectrometer. Rapid Commun. Mass Spectrom. 2002, 16, 512-526.

13. Javahery, G.; Thomson, B. A. A Segmented RadiofrequencyOnly Quadrupole Collision Cell for Measurements of Ion Collision Cross Section on a Triple Quadrupole Mass Spectrometer. J. Am. Soc. Mass Spectrom. 1997, 8, 697-702.

14. Dawson, P. H. Quadrupole Mass Spectrometry and Its Applications. AIP Press: Woodbury, 1995; pp 19-24.

15. March, R. E. An Introduction to Quadrupole Ion Trap Mass Spectrometry. J. Mass Spectrom. 1997, 32, 351-369.

16. Dehmelt, H. G. Radiofrequency Spectroscopy of Stored Ions I: Storage. Adv. Atomic Mol. Phys. 1967, 3, 53-72.

17. Major, F. G.; Dehmelt, H. G. Exchange-Collision Technique for the rf Spectroscopy of Stored Ions. Phys. Rev. 1968, 170, 91-107.

18. Press, W. H.; Teukolsky, S. A.; Vetterling, W. T.; Flannery, B. P. Numerical Recipes in C, 2nd ed.; Cambridge University Press: London, 1992; Sec 16.4, p 724.
19. Arfken, G. Mathematical Methods for Physicists. Academic: New York, 1968; p 530.

20. Douglas, D. J.; Glebova, T. A.; Konenkov, N. V.; Sudakov, M.Y. Spatial Harmonics of the Field in a Quadrupole Mass Filter with Circular Electrodes. Tech. Phys. 1999, 44, 1215-1219.

21. Schulte, J.; Shevchenko, P. V.; Radchik, A. V. Nonlinear Field Effects in Quadrupole Mass Filters. Rev. Sci. Instrum. 1999, 70, 3566-3571.

22. Gerlich, D. Inhomogeneous RF Fields: A Versatile Tool for the Study of Processes with Slow Ions. In State-Selected and Stateto-State Ion-Molecule Reaction Dynamics, Part 1: Experiment; John Wiley and Sons, Inc. 1992; Chap LXXXII, pp 1-176.

23. Franzen, J.; Gabling, R.-H.; Schubert, M.; Wang, Y.; Nonlinear Ion Traps. Practical Aspects of Ion Trap Mass Spectrometry; Vol. I; CRC Press, Inc.: 1995; pp 49-167.

24. Tolmachev, A. V.; Chernushevich, I. V.; Dodonov, A. F.; Standing, K. G. A Collisional Focusing Ion Guide for Coupling an Atmospheric Pressure Ion Source to a Mass Spectrometer. Nucl. Instrum. Methods Phys. Res. B 1997, 124, 112-119.

25. Covey, T.; Douglas, D. J. Collision Cross Sections for Protein Ions. J. Am. Soc. Mass Spectrom. 1993, 4, 616-623.

26. Flanagan, J. M. Mass Spectrometry Calibration Using Homogeneously Substituted Fluorinated Triazatriphosphorines. U.S. Patent 5,872,357, 1999.

27. Schwartz, J. C.; Senko, M. W.; Syka, J. E. P. A Two-Dimensional Quadrupole Ion Trap Mass Spectrometer. J. Am. Soc. Mass Spectrom. 2002, 13, 659-669.

28. Splendore, M.; Londry, F. A.; March, R. E.; Morrison, R. J. S.; Perrier, P.; Andre, J. A Simulation Study of Ion Kinetic Energies During Resonant Excitation in a Stretched Ion Trap. Int. J. Mass Spetrom. Ion Processes 1996, 156, 11-29. 